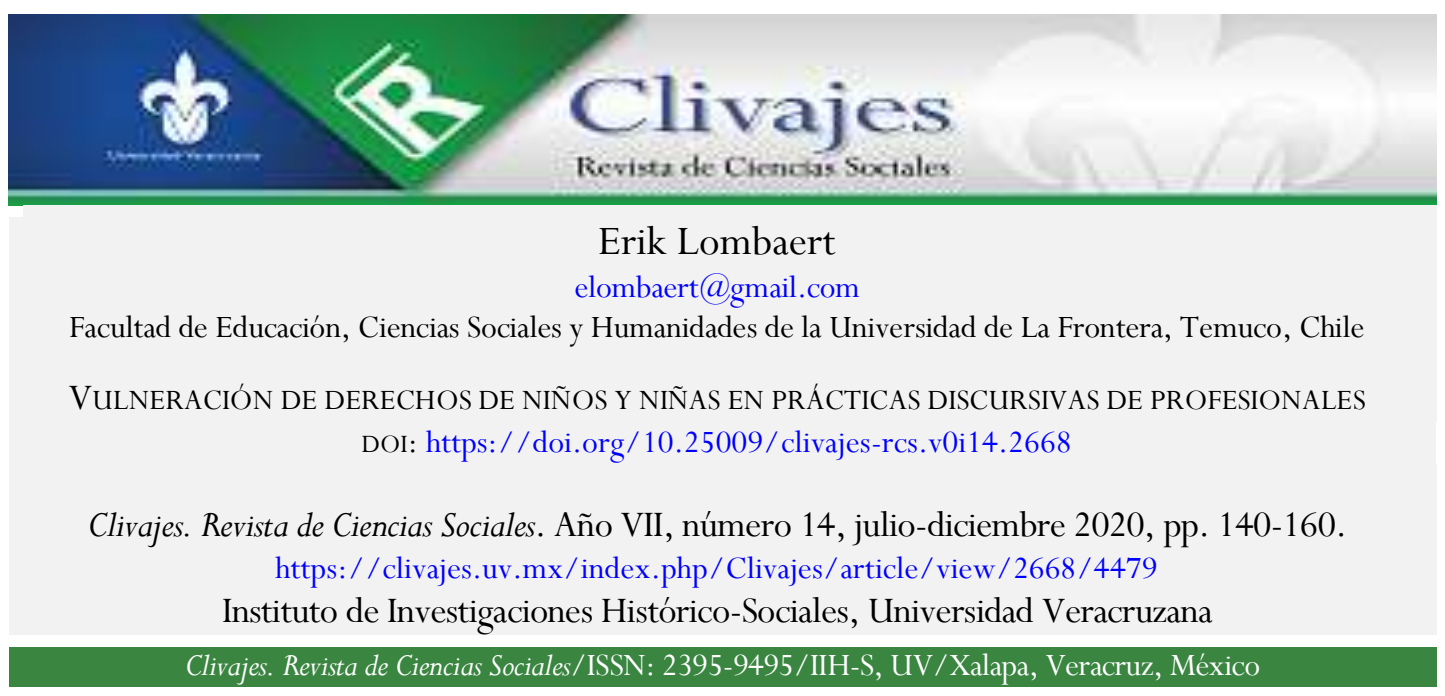

Recibido: 11/04/2020

Aceptado: 20/05/2019

Dictaminado: 08/09/2020 


\title{
VULNERACIÓN DE DERECHOS DE NIÑOS Y NIÑAS EN PRÁCTICAS DISCURSIVAS DE PROFESIONALES
}

\author{
Erik Lombaert ${ }^{*}$
}

\begin{abstract}
Resumen
Este artículo da cuenta de hallazgos de investigación basada en el análisis crítico de discursos de equipos de profesionales de los sectores de Justicia, Salud y Educación en la Región Metropolitana y La Araucanía de Chile, que atienden a niños, niñas y adolescentes en condiciones de vulnerabilidad, con el propósito de explorar prácticas discursivas relativas a la judicialización de problemáticas que les afectan. Se develan diversas formaciones discursivas, de las cuales se describe el uso transversal y estratégico del enunciado "vulneración de derechos" en los espacios intersticiales, el cual caracteriza el campo de la protección especial y el reducido margen de discrecionalidad profesional, que permite y obliga la tensión entre el derecho fundamental de acceso a la justicia y la institución de la obligatoriedad legal y administrativa de denunciar situaciones meritorias de medidas judiciales de protección.
\end{abstract}

Palabras clave: Derechos del niño, Protección de la infancia, Agenciamiento, Discurso

\section{VIOLATION OF THE RIGHTS OF BOYS AND GIRLS IN DISCURSIVE PRACTICES OF PROFESSIONALS}

\begin{abstract}
Summary
This article reports on research findings based on the critical analysis of speeches by teams of professionals from the Justice, Health and Education sectors in the Metropolitan Region and La Araucanía of Chile, who care for children and adolescents in vulnerable conditions, with the purpose of exploring discursive practices related to the prosecution of problems that affect them. Various discursive formations are revealed, of which the transversal and strategic use of the statement "violation of rights" in interstitial spaces is described, which characterizes the field of special protection and the reduced margin of professional discretion, which allows and obliges the tension between the fundamental right of access to justice and the institution of the legal and administrative obligation to report situations worthy of judicial protection measures.
\end{abstract}

Keywords: Children's rights, Child protection, Agency, Speech

\section{VULNÉRABILITÉ DES DROITS DES GARÇONS ET DES FILLES DANS DES PRATIQUES DISCURSIVES DES PROFESSIONNELS}

\section{Résumé}

Dans cet article on rend compte des trouvailles de recherche basée dans l'analyse critique des discours d'équipes de professionnels des secteurs de Justice, Santé et Éducation dans la Région Métropolitaine et La Araucanía au Chile, qui s'occupent des garçons et des filles en conditions de vulnérabilité, au but d'explorer de pratiques discursives relatives à la judiciarisation des problématiques qui leur concernent. On dévoile diverses formations discursives, desquelles on décrit l'utilisation transversale et stratégique de la phrase «vulnérabilité des droits» dans les espaces interstitiels, laquelle caractérise le champ de la protection spéciale et la marge réduite de discrétion professionnelle qui permet et oblige la tension entre le droit fondamental d'accès à la justice et l'institution de l'obligation légale et administrative de dénoncer des situations méritoires des mesures judiciaires de protection.

Mots clés : Droits de l'enfant, Protection de l'enfance, Appropriation, Discours

\footnotetext{
* Magister en Ciencias Sociales Aplicadas (Universidad de La Frontera de Temuco, Chile), abogado (Universidad Católica de Lovaina, Bélgica) y trabajador social (Universidad Academia de Humanismo Cristiano de Santiago de Chile), actualmente adscrito a la Facultad de Educación, Ciencias Sociales y Humanidades de la Universidad de La Frontera de Temuco, Chile. Contacto: elombaert@gmail.com.
} 


\section{INTRODUCCIÓN}

Los profesionales que trabajan en el ámbito de la niñez y la adolescencia en contextos de vulnerabilidad, a saber, procedentes de sectores que se caracterizan por la desigualdad de condiciones y expectativas de vida, ya sea en el campo de la protección especial, de la salud o de la educación, se enfrentan, con frecuencia, a situaciones de abuso sexual o laboral; de maltrato, violencia intrafamiliar, abandono, negligencia, permanencia prolongada en la calle, bullying, deserción escolar y consumo problemático o no- de alcohol u otras sustancias ilícitas, así como a conductas disruptivas y desafiantes hacia la autoridad, por nombrar sólo algunas. A estos profesionales, les corresponde decidir, actuar o no, informar a los Tribunales de Justicia sobre estas situaciones o conductas, acuñar procedimientos administrativos, valorar, motivar o desincentivar que los involucrados resuelvan por iniciativa propia o elijan estrategias alternativas de resolución de conflictos, lo cual implica un posicionamiento al respecto, observando el principio de acceso a la justicia, ${ }^{1}$ a la vez que la obligatoriedad ${ }^{2}$ legal y administrativa, vigente en Chile, para judicializar situaciones con alcance penal y meritorio de medidas judiciales de protección. Supone, por tanto, la toma de decisiones basadas en pre-juicios, o sea, en juicios previo a la deliberación de las instancias formalmente instituidas y facultadas para dirimir si las "situaciones" efectivamente son constitutivas de delito o merecedoras de una medida judicial (Lombaert, 2014).

Sin embargo, estas decisiones no sólo involucran consideraciones normativas y éticas o deontológicas, ni son exclusivamente producto de elaboraciones racionales: se ven afectadas por mandatos institucionales de dependencia; exigencias patronales o de otra índole, propias del proyecto o programa de participación, puesto organizacional y equipo de trabajo; recursos aprovechables, financieros, reticulares u otros; presiones mediáticas; interpretaciones acordes con el "régimen de mirada" disciplinario (Matus Sepúlveda, 1999, p. 97), de -a veces- escasa o, al menos, insuficiente información disponible o alcanzable en plazos más o menos acotados, etc.

\footnotetext{
${ }^{1}$ Artículo $19^{\circ} \mathrm{N}^{\circ} 3$ de la Constitución Política de la República de Chile, además del artículo $20^{\circ}$ de la misma Constitución; Artículo $8^{\circ}$ (Derecho a las garantías judiciales) y $25^{\circ}$ (Derecho a la protección judicial) de la Convención Americana de los Derechos Humanos "Pacto San José de Costa Rica" (1969); Artículo 14 del Pacto Internacional de Derechos Civiles y Políticos (1966).

${ }^{2}$ Entre otros, Art. 175 de la Ley No 20.074 que establece el Código Procesal Penal; Art. 61, literal k) del DFL No 29 del Ministerio de Hacienda que fija texto refundido, coordinado y sistematizado de la Ley N 18.834 , sobre Estatuto Administrativo; Art. $84^{\circ}$ de la Ley $\mathrm{N}^{\circ} 19.968$ que crea los Tribunales de Familia; Art. 14 ${ }^{\circ}$ de la Ley 20.032 que establece el sistema de atención a la niñez y adolescencia a través de la red de colaboradores del SENAME y su régimen de subvención; Art. $13^{\circ}$ de la Ley $\mathrm{N}^{\circ} 20.000$ que sustituye la Ley $\mathrm{N}^{\circ} 19.366$ que sanciona el tráfico ilícito de estupefacientes y sustancias sicotrópicas.
} 
Por cierto, sin pretender exhaustividad, a esta "poli-demanda institucional" (Schöngut Grollmus, 2017, p. 42) deben agregarse otras dimensiones, como los sistemas de significaciones, creencias, motivos y motivaciones de la o las comunidades y sus sentidos de pertenencia y referencia, así como las expectativas, opiniones y actitudes de los propios niños, niñas y adolescentes; las de sus familiares, adultos responsables de su cuidado y crianza, y de sus comunidades de pertenencia.

No obstante, en la práctica profesional se observa una preferencia por soluciones judiciales, en desmedro de alternativas de gestión de los problemas referidos, pese a la vigencia de la Convención de los Derechos del Niño ${ }^{3}$ y otros tratados internacionales de derechos humanos, que sugieren respuestas colaborativas y administrativas efectivas por sobre la judicialización de las necesidades de niños, niñas y adolescentes, y de los conflictos que les involucren -incluso en caso de alegar, acusar o declarar culpable [a alguno] de haber infringido las leyes penales (Naciones Unidas, 1989: Art. 40\% b; 1990)- ${ }^{4}$ y a pesar también de que diversos autores (Agamben, 2004; Daich, 2004; Eirás Nordenstahl, 2005; Neuman, 2005; Nicolini, 2011; Pesqueira Leal, 2005) califican la judicialización como la desapropiación de la contienda de los involucrados y su traspaso al árbitro de la judicatura, lo cual no siempre, ni necesariamente, es la mejor alternativa de resolución de conflictos. En su respuesta a la "solicitud de investigación [presentada el 22 de junio de 2015] en relación a la situación de niños, niñas y adolescentes (NNA) privados de entorno familiar que se encuentran en Centros Residenciales de Chile, bajo control directo o indirecto del Servicio Nacional de Menores (SENAME)", el propio Comité de los Derechos del Niño (2018) indicó como una de las causas estructurales "que han favorecido las graves y sistemáticas violaciones" del Estado: "la excesiva judicialización del sistema" (pp. 3 y 122).

La preferencia por la judicialización parece ir en paralelo con la paulatina ampliación institucional de la obligatoriedad de denunciar situaciones de vulneración de derechos con alcance penal y meritorias de medidas judiciales de protección - presente en la legislación e instrucción administrativa con respecto al ámbito de la protección especial de la niñez y la adolescencia en Chile--, y con la escasez de mecanismos de protección de derechos de niños, niñas y adolescentes en sede administrativa o de modo colaborativo.

\footnotetext{
${ }^{3}$ En adelante "la Convención”, “CDN" o "Naciones Unidas, 1989".

${ }^{4}$ No obstante que nos referimos específicamente al niño y la niña "de quien se alegue que ha infringido las leyes penales o a quien se acuse o declare culpable de haber infringido esas leyes”, el carácter fundamental de interdependencia e indivisibilidad de los derechos hace extensivo dicho estipulado a todos los niños, las niñas y los adolescentes.
} 
Es en este escenario se inscribe "La judicialización de conflictos interaccionales que involucran a niños, niñas y adolescentes en las prácticas discursivas de profesionales", una investigación que precisamente nos permitió indagar en las prácticas discursivas de profesionales de los sectores de justicia, educación y salud, provenientes de espacios altamente urbanizados de la Región Metropolitana y zonas rurales de La Araucanía de Chile, que trabajan en el ámbito de la niñez y la adolescencia en condiciones de vulnerabilidad, específicamente en el momento de calificar los problemas sobre los que intervienen y de la planificación del curso de acciones a emprender. ${ }^{5}$

Durante la investigación, además de describir las constelaciones relacionales propias de dicho ámbito, referidas por los participantes del estudio, examinamos los discursos producidos por profesionales en los procesos de toma de decisiones sobre la pertinencia de la judicialización de las problemáticas que les tocaba atender; destacan las formaciones discursivas "vulneración de derechos", "integralidad" y “confidencialidad”. En este artículo, se profundizará en algunas de las aplicaciones discursivas del primer enunciado.

Entre los hallazgos del estudio, se concluye un uso estratégico de la etiqueta en el campo de la protección especial de los derechos de la niñez y la adolescencia, caracterizado como "intersticial” (Bhabha, 2013, cit. por Cabello Valenzuela \& Palominos, 2018, p. 84) y con espacios reducidos de agenciamiento o maniobrabilidad (Magistris, 2018) que permiten y obligan la tensión entre el derecho de acceso a la justicia y la obligatoriedad de denunciar situaciones meritorias de medidas judiciales de protección.

\section{PRÁCTICAS DISCURSIVAS Y ENUNCIADOS}

La noción de prácticas discursivas es central para la investigación. Éstas se postulan como actos de enunciación y argumentación que no sólo transmiten información, portan significaciones e intencionalidades o sentidos, sino también acompañan e incitan a la acción, la constituyen e instituyen: son más que actos de habla, son acontecimientos de comunicación no exclusivamente verbales (Habermas, 1999); se postulan como formaciones discursivas (Foucault, 2013) que actualizan, integran, diferencian y

\footnotetext{
${ }^{5}$ Este artículo se basa, precisamente, en "La judicialización de conflictos interaccionales que involucran a niños, niñas y adolescentes en las prácticas discursivas de profesionales”, el trabajo de investigación (2014-2016) presentado por el autor para obtener el título de Magister en Ciencias Sociales Aplicadas, con financiamiento de CONICYT (2014/2015), bajo la dirección académica del Dr. Ricardo Pérez-Luco.
} 
regularizan constelaciones de singularidades (Deleuze, 2014, p. 12; 2015, p. 234), de fuerzas singulares encarnadas en enunciados; se inscriben en trayectorias de formaciones enunciativas o de diagramas de fuerzas, que se caracterizan por afectar y ser afectadas por otras.

En este entramado, las prácticas discursivas devienen fuerzas que se resisten: son polos que afectan o inciden no sólo sobre otros, sino también sobre sí mismos; se inscriben, se relacionan e interactúan con trayectorias enunciativas: las prácticas discursivas las construyen y reconstruyen, las confirman o interpelan, las refuerzan y modifican; articulan, a la vez que obedecen al "conjunto de reglas anónimas, históricas, siempre determinadas en el tiempo y el espacio que han definido en una época dada, y para un área social, económica, geográfica o lingüística dada, las condiciones de ejercicio de la función enunciativa" (Foucault, 1970, p. 198) que crean y recrean realidades capaces de abrazar, acompañar, impugnar y refrendar procesos de acción. A la par, estos últimos invocan, legitiman, autorizan, invalidan, o al menos interpelan, lo enunciado. Son prácticas constituyentes de enunciados, o sea, formativas de realidades discursivas, que se diferencian de las visibilidades, las realidades fácticas o los procesos. Los procesos (las visibilidades) y el proceder (las enunciaciones) constituyen procedimientos (Deleuze, 2015, p. 34).

El acontecimiento enunciativo implica la selección de modalidades de comunicación y conceptos anclados en doxas y teorías, sabidurías y epistemes, para hablar sobre "algo", sobre un "tema"; cada una de estas selecciones invoca, y a la vez revoca, formaciones enunciativas previas y contemporáneas, situadas temporoespacialmente. Los discursos - asumidos como prácticas- forman sistemáticamente los objetos de que hablan (Foucault, 1970, p. 68), sea explícita o implícitamente. En tal sentido, interesa la relación entre las prácticas discursivas y el contexto sociocultural o ideológico (Orlandi, 2012), "las condiciones de su emergencia, sus reglas de formación, las estrategias de las que da cuenta, sus efectos de poder" (Botticelli, 2011, p. 118), su legitimidad, siempre situada temporo-espacialmente.

Las prácticas discursivas establecen y re-establecen, y, como tales, (de)velan relaciones no sólo entre textos, sino también entre actores. Implican un posicionamiento en el entramado relacional social y societal. Los enunciados derivados de estas prácticas transitan entre diversos campos de fuerzas. No están necesariamente a la vista. Son una especie de común denominador, despejado de las particularidades de cada campo. Sus regularidades deben ser extraídas de las prácticas discursivas del campo de relaciones de fuerzas y resistencias de observación. 


\section{PRÁCTICAS DISCURSIVAS PROFESIONALES}

Soler y Flecha (2010) postulan los discursos, en cuanto prácticas, como acciones enunciativas diferenciadas según el "predominio de las interacciones de poder surgidas de la propia intencionalidad del hablante y/o de las desigualdades de la estructura social” (p. 373). Estas desigualdades se reflejan de modo particular en el discurso institucional, como modalidad enunciativa que, en particular, refiere acciones comunicativas entre participantes asimétricamente relacionados con diferencias de "estatus interaccional”, en el sentido de "capacidad de control discursivo" y "oportunidad de intervención" (Prego Vázquez, 1998, p. 266).

Las prácticas discursivas entre profesionales responsables de la implementación y ejecución de políticas, programas, proyectos y acciones, entre éstos y sus jerarcas, y entre ellos y sus "clientes", "usuarios", “destinatarios" o "participantes" - como sea que les denominen-, están determinadas, o al menos constreñidas, por sus posiciones en el entramado institucional, así como por los mandatos, deberes y derechos asociados (Carmona García, 2009, pp. 29-30). Por cierto, las mencionadas asimetrías varían, según el tipo de institucionalidad y la ubicación jerárquica de sus representantes, delegados o voceros.

En el mismo tenor, la intervención social aludida y de interés para el presente artículo es una actividad profesional y, por tanto, laboral, o sea, dependiente y remunerada, a la vez que inscrita en las políticas públicas de carácter social y sectorial. Ello significa una jerarquización de mandatos desde las autoridades de mayor jerarquía, hasta las entidades encargadas de su implementación en el aparato estatal, e involucra, luego, a los equipos ejecutores de las acciones derivadas, sean éstas parte del ámbito estatal -administración directa, municipal u otra- o del mundo del derecho privado por asignación directa, vía licitatoria u otros mecanismos, estableciendo una suerte de “contratos" entre cada peldaño de este engranaje.

En este eslabonamiento de "contratos", según la precisión de sus contenidos, las modalidades de seguimiento y las facultades y capacidades de fiscalización, entre otros factores, se realizan formulaciones y reformulaciones, progresivamente operativas, de las metas y los resultados esperados (de las "intenciones" de la intervención). Los últimos, en este proceso, son los profesionales, de cara a los "destinatarios" finales, quienes traducen en acciones los mandatos, las demandas, las necesidades, las expectativas y perspectivas de - en este caso- niños, niñas, adolescentes, sus familias, sus colectivos de pertenencia e, inclusive, de la "opinión pública”, o ciudadanía en su expresión genérica, y de sus 
propias comunidades de sentido de pertenencia, siempre desde el acervo familiarizado de experiencias, según el momento biográfico particular tanto del equipo de trabajo como de cada uno de sus integrantes.

Tomando en cuenta que, entre otras, las relaciones de jerarquías societales no sólo están sujetas a presiones "desde arriba", sino que inciden también "hacia arriba" de la pirámide de dependencia institucional, las prácticas de los profesionales incitan a la actualización de procedimientos, es decir, de cometidos enunciativos y cursos de acción. Estas prácticas se forjan en las relaciones de fuerza al interior de cada equipo con la institución de dependencia -sea organizacional, financiera o técnicamente--, los "destinatarios", "usuarios", “participantes" o "pacientes" - personas, familias, grupos, organizaciones, comunidades-, los colectivos, las agrupaciones, las instituciones, los servicios, afines o no, que comparten la misma temática y/o territorio de inserción, con los Tribunales de Justicia y la opinión pública, entre otras instancias. Todos ellos, actores interrelacionados e interconectados, con diferentes niveles de autonomía, lo cual remite a la comprensión de las relaciones de poder como relaciones de fuerza, o sea, de agenciamiento o estrategización ya no unidireccional, sino bi, o mejor aún, multidireccional. Las constelaciones de relaciones de fuerza subyacen a y son actualizadas en las prácticas discursivas.

\section{Aspectos METODOLÓGICOS}

Para el estudio al cual remite este trabajo, se configuraron dos corpus muestrales: 1) Discursos producidos por profesionales a través de la técnica de grupos de discusión, y 2) Textos legales y administrativos. El primer corpus reúne discursos de profesionales de equipos que atienden a niños, niñas y adolescentes de tres diferentes sectores (justicia, salud y educación) y dos localidades (la urbe metropolitana y localidades precordilleranas de La Araucanía). Para lograr mayor heterogeneidad, optamos por el muestreo de casos (prácticas discursivas de equipos de profesionales específicos) con máxima variación o heterogeneidad entre grupos (Valles, 1999, p. 313), asumiendo los criterios de localidad y ámbito sectorial de adscripción.

Para la composición de los grupos, preferimos la integración de los profesionales que habitualmente participan de las discusiones previas y la toma de decisiones con respecto al curso de intervención para o desde las y los niños y adolescentes que corresponde atender, independientemente de la estructuración del equipo de trabajo o de su funcionamiento interdisciplinario. En caso de que las discusiones previas y/o la 
toma de decisiones fueran individuales (con o sin consulta previa a uno u otro par, experto o autoridad, de acuerdo con las normas protocolarias preestablecidas o los hábitos no formalizados u ocasionales), se intentó conformar el grupo con dichos profesionales y sus respectivos consultantes, supervisores o tomadores de las mencionadas decisiones. En tal sentido, se buscó la máxima heterogeneidad intragrupal (Valles, 1999, p. 313).

Ahora bien, para la producción de las prácticas discursivas se aplicó la técnica de “grupos de discusión” de modo "autosuficiente”, es decir, se usó la técnica por sí sola, como modo "autocontenido" (Valles, 1999, pp. 301-302), con ocho equipos, en lo que se involucró un total de 42 profesionales. Del sector Justicia, participaron los equipos de dos Oficinas de Protección de Derechos de Niños, Niñas y Adolescentes (OPD) y dos Programas de Prevención Focalizada (PPF), ambas modalidades de intervención, subvencionadas por el Servicio Nacional de Menores (SENAME); del sector Salud, participaron dos equipos de salud mental y del sector Educación, grupos de profesionales de dos escuelas básicas; en cada ocasión, uno de la Región Metropolitana y otro de La Araucanía.

Las conversaciones grupales se realizaron con grupos operativos con cierta tradición de trabajo y en las dependencias habituales de trabajo en común. De este modo, pretendimos cercanía a la "entrevista grupal de campo", "natural” o "en situación”, en el ambiente propio de trabajo, como simulacro de una reunión de trabajo común, en el día a día laboral (Valles, 1999, p. 293): "que refleja sus habituales estereotipos y discusiones" (Villasante, 1999, p. 418), acercándonos a la técnica de observaciónparticipante. El guion para la discusión grupal incluyó la invitación a analizar una situación teórico-hipotética que se presentó de igual forma a todos los equipos participantes. Tras el análisis de la información producida, se realizó un segundo encuentro con cada uno de los grupos de discusión, con la intención de que participaran los mismos actores, para devolver la información y recibir comentarios, observaciones y correcciones. De esta manera, buscamos un mayor acercamiento a las técnicas de producción de información de tipo participativo (Valles, 1999, p. 295).

El segundo corpus consistía en un conjunto de textos formales, de carácter público (leyes, decretos, bases, normas y orientaciones técnicas, etc.), que rigen los distintos dispositivos institucionales y programáticos de los tres sectores considerados en términos jurídicos y administrativos. Contienen definiciones, normas, mandatos, instrucciones, protocolos, orientaciones, planes, entre otros. 
Para el análisis de ambos corpus de discursos, no nos propusimos examinar aspectos sintácticos ni semánticos, sino realizar una escucha, lectura, interpretación y comprensión de las formaciones discursivas de relevancia $\mathrm{y}$, específicamente, de su producción en el entramado institucional o, con mayor amplitud, societal. Buscamos develar argumentos que fundan y constituyen la calificación de los problemas a intervenir y, en particular, la opción por la judicialización o la gestión de alternativas de resolución de conflictos. No pretendimos aislar o tipificar prácticas, en este caso discursivas, para cada uno de los nodos o intersecciones del cruce perpendicular de la sectorización (justicia, salud y educación) y la localización (urbe metropolitana v/s sectores rurales de La Araucanía). Más que buscar las diferencias sectorializadas y localizadas, o sea, lo "típico", "propio" o "inherente" de cada sector y lugar (segmentación), importaba observar los enunciados que transitan entre-nodos y analizar los conceptos discursivos "en el cruce de los sistemas homogéneos por los cuales pasa el enunciado" (Deleuze, 2015, p. 141).

En un primer momento, se analizaron los corpus de discursos, infiriendo sus contenidos, identificando los "sobre quién" (actor) o "sobre qué" (acción) se habla y sus respectivos opuestos (quién o qué se excluye explícita o implícitamente). Para cada sentencia o segmento de discurso, se indagó en los "porqué" (desde qué lugar) y el "para qué" (con qué objeto/objetivo) de su empleo, los juicios que albergaban y las emociones que los acompañaba, considerando su distribución temporo-espacial, o sea, la cercanía o lejanía de lo aludido en relación con los oradores en tiempo y espacio. En un segundo momento, se realizó una revisión transversal (entre sectores, localidades y corpus muestrales) del uso de los enunciados más relevantes en relación con el objetivo del estudio, estableciendo relaciones entre los diversos contextos de su empleo (actores/acciones), concepciones, así como de sus respectivos opuestos, valoraciones (juicios/emociones) y lugar espacio-temporal, creando patrones y redes entre sus aplicaciones sectoriales, locales y muestrales).

En las siguientes líneas, exponemos sólo los resultados del proceso de análisis sobre la etiqueta "vulneración de derechos" y sus derivados. Cabe mencionar que la referida investigación se desarrolló de acuerdo con protocolos aprobados previamente por el Comité Ético Científico de la Universidad de La Frontera, lo que incluye el consentimiento informado de los y las participantes, con respecto a los objetivos y procedimientos del estudio, tipo de participación, su carácter voluntario, confidencialidad, uso y resguardo de los datos, entre otros aspectos. 


\section{Resultados}

La investigación sugiere la articulación del campo de acción de interés, como un intersticio societal con amplia diversidad de actores que colaboran y distraen intermitentemente en la consecución de los propósitos de los equipos profesionales protagonistas. En esta constelación de relaciones de fuerza, se observan prácticas de resistencia o agenciamiento; se actualizan instituciones enunciativas en su aplicación estratégica; operan y son operadas como herramientas de incidencia sobre otros actores y también como herramientas de ajuste a normas, a la vez que como refugios y escudos. Así se constata una apropiación estratégica (Cabello Valenzuela \& Palominos, 2018; De Certeau, 2000) de éstas.

Al respecto, se retiene la expresión "vulneración de derechos" como la más emblemática: se presenta con diversas aplicaciones y destaca su calificación como "vulneración grave", "de alta complejidad", "constitutiva de delito", "sujeta a la obligatoriedad de denuncia ante los Tribunales de Justicia”, etc., en relación con situaciones de abuso sexual, maltrato físico y psicológico, negligencia en el cuidado y la responsabilidad de protección por parte de los padres o de quienes asuman ese rol, entre otras, en desmedro de vulneraciones de otros derechos -políticos, económicos, sociales y culturales- que apenas se mencionan. Así, para nombrar sólo algunas, la expresión aludida no parece contener las violaciones al derecho a la participación, incluyendo el derecho a ser oído durante la defensa en juicio, y a la no discriminación por pertenecer a grupos minoritarios, situaciones todas que parecen no estar incluidas en el "ámbito de atención” o “de intervención” por ninguno de los equipos participantes en el estudio. Además, en la actualidad no se observan mecanismos legales genéricos por ejemplo, una Ley de Protección Integral de los Derechos de la Niñez- ni de protección específicos - por ejemplo, una defensoría legal de la niñez (Lathrop Gómez, Berrios \& Cillero, 2018).

A grandes rasgos, es posible constelar dos formaciones enunciativas: una amplia y otra restrictiva. En su sentido amplio, la "vulneración de derechos" se relaciona con el denominado "enfoque de derechos", un instrumento conceptual para el diagnóstico, el diseño, la programación y la evaluación de la intervención social, con los Derechos Humanos - en este caso de la niñez y adolescencia- como estándar o medida de referencia; cada situación que no cumple con tal referente es considerada "vulneración de derechos". En su sentido restringido, la expresión refiere una situación constitutiva de delito $\mathrm{y}$, por tanto, obligatoriamente sujeta a la judicialización. Podría postularse 
que entre ambos extremos existe una amplia gama de variaciones; entre ellas, aunque muy cercanas a la lectura restrictiva del concepto, se cuentan las situaciones, consideradas no constitutivas de delitos, pero susceptibles de medidas judiciales de protección sujetas al arbitrio de los Tribunales de Familia.

Ambas comprensiones, la amplia y la restrictiva, pueden ubicarse en los extremos de un continuum entre los que se sostiene una graduación de la misma concepción. Se trata de "bipolaridades, (...) oposiciones sustanciales en un campo de fuerzas recorrido por tensiones polares que están presentes en cada uno de los puntos sin que exista posibilidad alguna de trazar líneas de demarcación” (Agamben, 2004, p. 12). Sin embargo, también podría postularse la tesis -aquí suscrita- de que ambas comprensiones de los vocablos "vulneración de derechos" constituyen formaciones enunciativas diferentes.

Mientras la concepción amplia, relacionada con el ya referido "enfoque de derechos", apela a cada situación que no pasa el examen de los estándares internacionales de los Derechos Humanos, de acuerdo con las condiciones sociales colectivas o consideradas aceptables para una sociedad determinada en el momento histórico más o menos acotado-, la concepción restrictiva apela a una situación específica, precisa y personalizada. Mientras la segunda refiere un conflicto interpersonal a ser mediado por un juez desde la perspectiva del derecho positivo, la primera refiere condiciones de vidas situadas (Bustelo Graffigna, 2005). Mientras la formación amplia remite a "formas-de-vida", la restringida, lo hace a la "nuda-vida" (Agamben, 2001; Lazo Briones, 2017). Mientras la primera sugiere una aplicación universal, la segunda se postula como focalizada.

Pese a que se observan múltiples referencias a la "integralidad" de las intervenciones de los equipos de profesionales participantes en la investigación, dicho enunciado deviene de la selección y focalización - no de la universalidad. Mientras la primera comprensión de la etiqueta "vulneración de derechos" - en su sentido ampliose ancla en la "doctrina de la protección universal e integral de derechos", la segunda su alter "restringido"- roza con la "doctrina de la irregularidad", supuestamente superada o en vías de superación, desde la entrada en vigencia de la CDN en Chile, situación contradicha por múltiples autores (Avello Saez, Román Morales \& Zambrano Constanzo, 2017; Estrada, 2018; Lathrop Gómez, 2014). Mientras la primera remite la tutela de derechos, la segunda se acerca a la tutela de personas o, al menos, -aunque no es lo mismo- a la tutela de derechos subjetivos. 
La primera se inscribe en el postulado de Bustelo (2005), en cuanto propone “todos los derechos de los niños/as y adolescentes [como] derechos «sociales» en el sentido de que su garantía es esencialmente política y, por lo tanto, corresponde a la sociedad en su conjunto implementar". Agrega que, como tales, son "heterónomos": "los derechos de niños/as y adolescentes son los derechos de «ellos», de aquellos que están más allá de mí, de aquellos que me sobrevivirán (...) son pura negación de nuestra 'mismidad', pura generosidad sin esperar reciprocidad” (pp. 264-265). Desde ahí, se enfatiza en la restitución de las garantías, sin perjuicio de la reparación del daño, lo que se aproxima a la tradición de la justicia restaurativa. De este modo, destaca la importancia de la autonomía de las infancias, a la vez que de su heteronomía en relación con la adultez, y del reconocimiento de la igualdad para posibilitar el diálogo (Birulés en Arendt, 2013, p. 22), en este caso, intergeneracional.

La segunda, la formación restrictiva, es una etiqueta entre varias que circulan para calificar los problemas que les toca atender a profesionales del ámbito. Pese a que se distinguen etiquetas, con más o menos arraigo sectorial restrictivo - por ejemplo, sobre "necesidades educativas especiales", en el sector educativo; "enfermedades", en el sector salud; "maltratos" y "abusos", en el sector justicia- o disciplinario - así, se tiende a tildar ciertas expresiones como más propias de la psicología, de la sociología, de la pedagogía, de la fonoaudiología-, éstas son aplicadas transversalmente.

Los calificativos se aplican de modo que las situaciones a atender se ajusten al ámbito de intervención, a los objetivos y las perspectivas de cada equipo, desde su lugar en el conjunto de modalidades o niveles de intervención de cada sector y localidad de adscripción, es decir, de manera que sean relevantes para estos equipos que autorizan y obligan a actuar. Lo que no se logra o no corresponde ajustar a las construcciones enunciativas del espectro de atención del equipo, no se atiende; eventualmente, se deriva. En ese sentido, el proceso de calificación de la problemática presentada opera como un mecanismo de selección y focalización de "destinatarios", "usuarios", "pacientes" o "participantes". Permite controlar los flujos de demanda hacia los proyectos, ya sea en relación con los mandatos y metas -especialmente las relacionadas con coberturas con incidencia directa o indirecta en el financiamiento y, por tanto, la sobrevivencia del proyecto-, o según las capacidades y competencias -a veces, conveniencias (para los destinatarios y el propio equipo)- de atención.

Mientras tanto, también se observan lecturas multidimensionales de las problemáticas que se presentan $\mathrm{y}$, por tanto, de las respuestas a gestionar, o sea, los dispositivos, esfuerzos y recursos a movilizar a través del trabajo intersectorial, la práctica 
de redes. Se tiende a superar el dar respuestas acotadas a las demandas y solicitudes específicas iniciales; el uso de etiquetas calificativas de éstas permite seleccionar los asuntos a atender y, asimismo, gestionar colaboraciones de otros actores, derivaciones, interconsultas, etc.

No obstante, el hecho de que la etiqueta "vulneración de derechos" tenga mayor arraigo en los equipos de profesionales a cargo de proyectos ligados al sector justicia (en particular, el SENAME), y sin perjuicio de que éstos empleen, a la par, otras etiquetas, esto no significa que su uso sea exclusivo de ese sector, ya que los equipos de salud y educación también la aplican, sea al momento del primer contacto con alguna situación que les toca atender, sea al recalificar la problemática, tras fallar las estrategias propias de carácter persuasivo. Sin desconocer que circula una amplia variedad de comprensiones, la enunciación, despojada de sus particularidades sectoriales, que transita predominantemente entre todos, es la que refiere a situaciones constitutivas de delito o meritorias de medidas de protección y, como tales, sujetas a la obligatoriedad legal de ser denunciadas. Empero, no necesariamente: también se emplea frente a situaciones en las cuales se considera importante el uso de la fuerza para contrarrestar o doblegar resistencias hacia el proyecto, el equipo o su propuesta de intervención, o para desnaturalizar situaciones de violencia hacia niños, niñas y adolescentes, generalmente al no lograr tal propósito con otras acciones a su alcance. En este mismo sentido, la etiqueta calificativa "vulneración de derechos" se aplica para forzar el acceso a ciertos servicios, ya porque la judicialización es un requisito de ingreso, ya porque no hay cupos, se niega que los haya o requieren ser logrados "a la fuerza”. A esto se agrega situaciones de sospecha de peligro para niños, niñas y adolescentes (primordialmente, sobre su integridad física) que no se logra despejar a partir de acciones voluntarias o persuasivas.

La calificación de problemas como "vulneración de derechos" implica una "mutación" enunciativa: Se transforma "en relevancias legales o, lo que es lo mismo, en cuestiones legalmente relevantes" (Daich, 2004, p. 355) y, por tanto, “judiciables" o "justiciables", independientemente de que luego se denuncie o se solicite una medida de protección ante la autoridad "judicial” correspondiente. "Las experiencias cotidianas no interesan en términos de lo que significan para los individuos sino que deben traducirse a otras formas para que se transformen en problemas [que] puedan ser procesados y digeridos por el sistema” (Smart, 1995, en Daich, 2004, p. 355), en este caso, judicial. Así, tildar un problema como "vulneración de derechos" (en su sentido estricto) conecta con el sector de justicia, en cuanto institucionalidad facultada para el 
uso legítimo de la fuerza. Al mismo tiempo, evitar la aplicación de la etiqueta permite prescindir de la derivación a y, por tanto, la intromisión de proyectos ligados al sector justicia.

En algunas oportunidades, los profesionales del sector de educación y, con mayor razón, del de salud, al calificar problemas que les toca atender como "graves vulneraciones de derechos" o al describirlos de modo que se ajusten a dicha categoría, solicitan directamente medidas de protección al Tribunal de Familia o denuncian los hechos ante las instancias policiales o la fiscalía correspondiente. Sin embargo, muchas veces derivan estos "casos" a instancias del SENAME, principalmente a las OPD, confundiendo estas instancias con entidades auxiliares de la judicatura o esperando que desde ahí los casos sean judicializados. En este contexto, la "derivación al SENAME” se equipara con una judicialización.

A la vez, se tiende a asociar la judicialización con separar a niños, niñas y adolescentes de sus familias de origen o con su "internamiento"-que, en este razonamiento reduccionista, se considera lo mismo- en alguna residencia administrada por el SENAME o por una de sus instituciones colaboradoras, lo cual no necesariamente se califica como una opción de protección o de "mejoría”, sino, en reiteradas ocasiones, como un "mal menor" e inclusive como una exposición a nuevas vulneraciones y un castigo. Este último razonamiento también se ha observado como argumento para evitar tal desenlace, o sea, al optar explícitamente por obviar el uso de la etiqueta "vulneración de derechos".

En tal sentido, sin desconocer que ambas construcciones discursivas -la "amplia" y la "restrictiva" - apelan a la judiciabilidad, el mero empleo de la restrictiva constituye en sí una judicialización, o sea, una confrontación, una coerción, el uso de la fuerza, de la violencia; busca, como hemos dicho, doblegar resistencias, desnaturalizar o desnormalizar el uso de la violencia, más allá o más acá de materializar la denuncia ante las autoridades penales o la solicitud de una medida de protección ante los Tribunales de Familia. Su mera aplicación tiene un efecto coercitivo. Al etiquetar los problemas como "vulneración de derechos" en su sentido restricto, la pregunta por la pertinencia de la judicialización se hace ineludible a tal nivel, que la no judicialización requiere de un fundamento - toda vez que no exista una protección de derechos alternativa a la judicial, sea pre-judicial, extra-judicial o simplemente no-judicial (realidad en el Chile de hoy)-, marcando de este modo una diferencia sustancial con la formación enunciativa aquí aludida como "vulneración de derechos" en su sentido amplio. 
Por cierto, ambas formaciones tienen en común la justiciabilidad, incluso la judiciabilidad -aunque la "vulneración de derechos" en su sentido amplio no parece ser considerada parte del ámbito de la protección especial de los derechos del niño (Lathrop et al., 2018), como ya hemos indicado--, miradas desde el derecho de acceso a la justicia como piedra angular de la matriz garantista. Sin embargo, en todos los equipos participantes en la investigación, independientemente de su posición sectorial y localización, observamos un énfasis en el uso de la formación restricta del concepto, sin desconocer ni contradecir su alter, mientras en las declaraciones institucionales, según el análisis del corpus muestral de documentos formales, se refiere siempre a su expresión amplia. Esta última es la versión oficial de las políticas públicas y de las políticas sociales sectorizadas, es decir, la versión presente en la retórica pública.

Para precisar, mientras el enunciado en su sentido restricto constituye una judicialización por su sola aplicación como etiqueta valorativa de la situación en observancia, su uso en sentido amplio sólo evoca la judiciabilidad como virtualidad (como "última ratio" y no como "último recurso"), invitando a la búsqueda de soluciones alternativas, sean de corte administrativo o colaborativo.

\section{DISCUSIÓN Y CONCLUSIONES}

A la pregunta por el interjuego entre prácticas discursivas y constelación de relaciones en el ámbito de la atención a niños, niñas y adolescentes que se encuentran en contextos de vulnerabilidad, se responde con el uso estratégico del enunciado "vulneración de derechos" al momento de enfrentar un escenario cada vez más complejo: no sólo se interactúa con otros actores, también se lidia con sugerencias, consultas, solicitudes, demandas, exigencias, orientaciones, indicaciones, reglas, mandatos, leyes, expectativas y perspectivas. Se debe cumplir con objetivos, resultados esperados, metas y productos; las acciones y sus resultados requieren de verificadores; existen protocolos, ritos, costumbres; y se enfrenta supervisiones, fiscalizaciones, controles de calidad y procesos de evaluación.

Este conjunto de referentes, de fuentes cada vez más diversas, es percibido muchas veces como incoherente y hasta incompatible a su interior. Cada referente comprende varios grados de obligatoriedad y observamos diferentes posicionamientos de los equipos de profesionales - los remitentes de este mosaico variopinto de mandatos-, incluso constatamos tensiones cruzadas al interior de algunos equipos. 
La aplicación de una u otra etiqueta calificativa de los problemas que los equipos de profesionales del ámbito considerado observan, no sólo permite y obliga la intervención, la evaluación, el diseño y la gestión del curso de acción, así como la colaboración con otros actores, sino también la activación de uno u otro protocolo. Así, tildar una situación como un problema de salud mental, por ejemplo, o como "vulneración de derechos" - etiquetas en absoluto no excluyentes- compromete procedimientos diferenciados. Etiquetar un problema como "vulneración de derechos" (en su sentido estricto) conecta con el sector de la justicia y, como hemos argumentado, implica una judicialización en sí, más allá o más acá de iniciar luego la denuncia o solicitud de una medida de protección ante la autoridad judicial. Sugiere una práctica estratégica.

En los discursos se observa un desplazamiento de "saberes" y "verdades" disciplinares - considerando que los participantes de la investigación son equipos de profesionales- hacia "saberes" y "verdades" normativo-administrativos, "relegando a un lugar secundario las implicancias de éstos para las personas sobre las que recaen sus servicios: niñas y niños, y las y los jóvenes" (Sola Morales \& Campos Garrido, 2019, p. 120). Aporta a la construcción niños, niñas y adolescentes "desubjetivados", sin agencia. En este contexto, surgen actualizaciones y usos estratégicos de formaciones enunciativas -aquí sólo destacamos la "vulneración de derechos"- que dan cuenta de agenciamientos por parte de los equipos de profesionales y de los "destinatarios", "usuarios" o "pacientes" de sus servicios - aspecto no desarrollado en este artículo.

En Chile no existe la protección especial en sede administrativa de los derechos del niño. Pese a ello -o quizás justamente por ello--, los equipos de profesionales han ido forjando un margen de agenciamiento que pareciera ampliar o al menos resguardar la discrecionalidad otorgada en el ordenamiento jurídico, administrativo y disciplinar con respecto a la obligación de denunciar situaciones de amenaza y vulneración grave de derechos, no constitutivas de delito. Con ello no se instituye una protección de tipo administrativo, sino que se habilita, por cierto, de modo indirecto y no precisa ni exclusivamente con dicha intención, la gestión de alternativas a la vía judicial para la resolución de conflictos.

En el caso de los equipos participantes de la investigación, esta construcción desde el margen (de la discrecionalidad formalmente autorizada) se construye - entre otras- en torno a la comprensión particular de la "vulneración de derechos", en su sentido restricto, y al uso estratégico de dicho calificativo de problemas que les son presentados o pesquisados. El paso al "enfoque de derechos", leído desde la doctrina de la protección 
integral de derechos - dejando pendiente la pretensión universalista-, con el dispositivo “vulneración de derechos" en su sentido amplio, se logra sólo de modo indirecto: con el uso estratégico de la etiqueta.

Desde el enfoque de derechos, en toda su amplitud, se observa que los derechos a garantizar, sujetos con frecuencia -inclusive sistemáticamente- a amenazas y vulneraciones - en todas las gradaciones de gravedad-, no se restringen a los civiles ni mucho menos a los que se relacionan con la integridad física, sino que a ellos se agrega los políticos, económicos, sociales, culturales y colectivos. Del mismo modo, la responsabilidad de la vulneración de cualquiera de estos derechos se extiende, por sobre los garantes interaccionales, al propio Estado, como garante principal, y a toda la sociedad, por cierto, con diferentes niveles de responsabilidad. En este sentido, suscribimos lo postulado por Bustelo con respecto al carácter político y social -más bien colectivo-de los Derechos del Niño.

En este contexto, sugerimos comprender la vulneración de derechos desde una perspectiva relacional, o sea, como un conflicto entre actores. Más allá y más acá de que muchos de estos conflictos alcancen el ámbito penal - y que, en este caso, corresponde la judicialización-, se asume que toda vulneración de derechos (no sólo los considerados como ámbitos de acción de los diversos dispositivos del área de protección) demanda su interrupción o la restitución de garantías, así como la prevención de que se repita, la reparación del daño sufrido y la restauración o recomposición de las relaciones sociales y societales comprometidas. Ello supera largamente la acción judicial del Estado.

Este planteamiento remite la problemática analizada a la necesidad de instituir la protección de los derechos fundamentales de la niñez y la adolescencia en sede administrativa y a reconocer la resolución alternativa (a la judicialización) de corte colaborativo. Sugiere, además, extraer del ámbito judicial las medidas de protección, restringiendo el rol de éste al control legal de las intervenciones -se propone que así seagestionadas en sede administrativa, devolviendo de este modo el protagonismo disciplinar a los equipos de profesionales, como instancias de intermediación en conflictos interaccionales que involucran a niños, niñas y adolescentes, reconociendo así su capacidad de agencia.

\section{REFERENCIAS}

Agamben, G. (2001). Medios sin fin. Notas sobre la política. Valencia: Pre-Textos. Agamben, G. (2004). Estado de Excepción. Buenos Aires: Adriana Hidalgo Editora. 
ARENDT, H. (2013). ¿Qué es la política? Barcelona: Arvato Services Iberia.

Avello Saez, D., Román Morales, A. \& Zambrano Constanzo, A. (2017). Abordaje de la intervención sociocomunitaria en programas de rehabilitación psicosocial: Un estudio de caso en dos dispositivos del sur de Chile. Psicoperspectivas. Individuo y Sociedad, 16(1), pp. 19-30. Recuperado de https://bit.ly/3qfGJux.

Botticelli, S. (2011). Prácticas discursivas. El abordaje del discurso en el pensamiento de Michel Foucault. Instantes y Azares. Escrituras nietzscheanas, 9, pp. 111-126. Recuperado de https: / / bit.ly/3sOU9zz.

Bustelo Graffigna, E. (2005). Infancia en Indefensión. Salud colectiva, 1, pp. 253-284. Recuperado de https: / / bit.ly/3rkIA2r.

Cabello Valenzuela, C. A. \& Palominos, S. (2018). Tácticas y estrategias de evasión del racismo cotidiano de trabajadores "negros" en Santiago de Chile. Chasqui. Revista Latinoamericana de Comunicación, 138, pp. 79-98.

Carmona García, J. P. (2009). Análisis de las prácticas discursivas en un centro de atención primaria: las interacciones de atención al usuario extranjero. Tesis Doctoral. Barcelona: Universitat Autònoma de Barcelona.

COMité de los Derechos del NiÑo. (2018). Informe de la investigación relacionada en Chile en virtud del artículo 13 del Protocolo facultativo de la Convención sobre Derechos del Niño relativo a un procedimiento de comunicaciones. $\mathrm{CRC} / \mathrm{C} / \mathrm{CHL} / \mathrm{INQ} / 1$. Naciones Unidas.

DAICH, D. (2004). Los procedimientos judiciales en los casos de violencia familiar. En Tiscordia, S. (Ed.), Burocracias y violencia. Estudios de antropología (pp. 327-379). Buenos Aires: Antropofagia.

De Certeau, M. (2000). La invención de lo cotidiano 1. Artes de hacer. (A. Pescador, Trad.). México: Universidad Iberoamericana.

Deleuze, G. (2014). El poder. Curso sobre Foucault. Tomo II (P. IRES \& S. PuENTE, Trads.). Buenos Aires, Argentina: Editorial Cactus.

Deleuze, G. (2015). El Saber. Curso sobre Foucault. Tomo I. (P. IRES \& S. PuENTE, Trads.). Buenos Aires: Editorial Cactus.

Eirás Nordenstahl, U. C. (2005). Mediación penal. De la práctica a la teoría. Buenos Aires: Librería-Editorial Histórica.

Estrada, F. (2018). Propuestas para la Reforma del Sistema de Protección. Santiago de Chile.

Foucault, M. (2013). La arqueología del saber (A. GARZÓn Del CAmino, Trad.) 2a ed. México/España/Argentina/Colombia: Siglo XXI Editores.

HABERMAS, J. (1999). Teoría de acción comunicativa I: Racionalidad de la acción y racionalización social. Madrid: Aguilar, Altea, Taurus, Alfaguara.

LATHRop GómeZ, F. (2014). La protección especial de Derechos de Niños, Niñas y Adolescentes en el Derecho Chileno. Revista Chilena de Derecho Privado, 22, pp. 197229. Recuperado de https: / / bit.ly/30gpJtv.

lathrop Gómez, F., Berrios, G. \& Cillero, M. (2018). Debate: Situación actual de los 
derechos de niños, niñas y adolescentes en Chile. Anuario de Derechos Humanos, 14, pp. 15-31. Recuperado de https://bit.ly/2OrItDD.

LAzO Briones, P. (2017). La resistencia intersticial y la crítica de la biopolítica en Giorgio Agamben. Revista Reflexiones Marginales, 39. Recuperado de https: / / bit.ly/3bhxtlg.

LOMBAERT, E. (2014). Complementariedad de la judicialización y el trabajo social. Niños, niñas y adolescentes amenazados, restringidos o vulnerados en el goce y ejercicio de sus derechos fundamentales. Saarbruck: Editorial Académica Española.

Magistris, G. P. (2018). La construcción del "niño como sujeto de derechos" y la agencia infantil en cuestión. Journal de Ciencias Sociales,11, pp. 6-28. Recuperado de https: / / bit.ly/3815iW0.

Matus Sepúlveda, T. (1999). Propuestas contemporáneas en Trabajo Social. Hacia una intervención polifónica. Buenos Aires: Editorial Espacio.

Matus SepúlvedA, T. (1990). Directrices de las Naciones Unidas para la prevención de la delincuencia juvenil (Directrices de Riad). Resolución 45/112 de la Asamblea General.

Neuman, E. (2005). Mediación penal (2 ${ }^{a}$ ed.). Buenos Aires: Universidad.

Nicolini, G. (2011). Judicialización de la vida familiar. Lectura desde el Trabajo Social. Buenos Aires: Espacio.

Organización de las Naciones Unidas. (1989). Convención sobre los Derechos del Niño. Resolución 44/25 de la Asamblea General.

Orlandi, E. (2012). Análisis de Discurso. Principios y procedimientos (E. Soto, Trad.). Santiago de Chile: Editorial LOM.

Pesqueira Leal, J. (2005). Mediación: Menores en riesgo e infractores en el contexto de la Seguridad Pública en México. México: Universidad de Sonora, Instituto de Mediación de México.

Prego VÁzquez, G. (1998). Algunas consideraciones sobre el discurso institucional. Interlingüística, 9, pp. 265-268. Recuperado de https:/ / bit.ly/305DUS7.

SchÖNGUT Grollmus, N. (2017). Ensamblajes socio-técnicos para la producción de intervenciones psicosociales en un programa de Servicio Nacional de Menores de Chile. Psicoperspectivas, 16(3), pp. 41-51. Recuperado de https: / / bit.ly/3c0ke82.

Sola Morales, S. \& Campos Garrido, N. A. (2019). Discurso estatal chileno en la protección de los derechos de niños(as) y jóvenes. Revista Latinoamericana de Ciencias Sociales, Niñez y Juventud, 17(1), pp. 105-124. Recuperado de https://bit.ly/3cequZE.

Soler, M. \& Flecha, R. (2010). Desde los actos de habla de Austin a los actos comunicativos: Perspectivas desde Searle, Habermas y CREA. Revista Signos, 43(2), pp. 363-375. Recuperado de https: / / bit.ly/3sHn9Jl.

VALlES, M. (1999). Técnicas cualitativas de investigación social: Reflexión metodológica y práctica profesional. Madrid: Síntesis. 
Villasante, T. (1999). De los movimientos sociales a las metodologías participativas. En Delgado, J. M. \& GutiérReZ, J. (Eds.), Métodos y técnicas cualitativas de investigación en ciencias sociales (pp. 399-424). Madrid: Síntesis. 